\title{
A NEW HIGH-INTENSITY ULTRASONIC TECHNOLOGY FOR FOOD DEHYDRATION *
}

\author{
J. A. Gallego-Juárez, G. Rodriguez-Corral J.C. Gálvez Moraleda, ${ }^{(1)}$ \\ T.S Yang ${ }^{(2)}$ \\ ${ }^{(1)}$ Instituto de Acústica, CSIC, Serrano, 144, 28006 Madrid \\ (2) Pinnacle Holding S.A. 19, Cité Bettenvies, L- 8479 EISCHEN, Luxembourg
}

Keywords and phrases: ultrasonic drying; ultrasonic dewatering

\begin{abstract}
At present, there is a growing interest in the field of dehydration for preserving food. Ultrasonic energy represents a means to obtain dehydration without affecting the food. This paper deals with an experimental study about the use of high-intensity ultrasound for vegetable dehydration by using a new power ultrasound generator and a procedure in which ultrasonic vibrations are applied in direct contact with the product and under a certain static pressure. The drying effect of this new process is compared with that obtained from forced-air drying assisted by air-borne ultrasonic radiation and from a conventional hot-air drying. The results show that by using the new technology and procedure it is possible to reduce dramatically the treatment time and it is relatively easy to reach a final moisture content in the samples less than $1 \%$. In addition, the
\end{abstract}


product qualities are well preserved, the sample rehydration is higher than $70 \%$ and, because of the high efficiency of the new ultrasonic system employed, the energy consumption is low. As a consequence of the results a new technology for industrial applications is under development.

\section{INTRODUCTION}

Dehydration is a method for preserving foods. Since the ancient sun-drying to the modern freeze-drying many methods have been explored to get demoisturization processes suitable to the requirements of the products to be treated.

There exist two basic conventional methods for the removal of moisture from a material: mechanical and thermal. Mechanical drying is based on pressing or centrifuging the material. In thermal drying the addition of whatever energy in the form of heat is required to evaporate the liquid. The first method removes moisture weakly attached while the second one can provide a more complete drying of the product.

The drying method suitable for each specific application is related with the attachment of the liquid with the solid material. In general, three types of attachment can be considered: chemical, mechanical and physicochemical. The last two types can be treated by conventional drying techniques. The mechanical attachment is that of porous materials containing wetting and capillary moisture. The physicochemical attachment is associated with adsorption and osmotic moisture. In capillary-porous materials both of these kinds of attachment are commonly present.

For food dehydration, the present conventional systems employ two main procedures: hot-air drying and freeze-drying. Hot-air drying is a widely used method but it can produce deteriorative changes in the food. Instead, in freeze-drying, where food pieces are first frozen and then ice sublimates, the product deterioration is negligible but the process is expensive.

High intensity sonic and ultrasonic waves have been used to increase the drying rate of materials. The acoustically assisted hot-air drying process permits the use of lower temperatures and may be useful for drying heat-sensitive materials [1, 2] 
When the material is completely covered with moisture and the evaporation takes place only at the surface it dries at a constant rate which depends on the diffusion through the surface of water vapour into the air, the moisture content, the relative velocity of the drying air and the surface temperature of the material [3]. The constant rate period of drying is followed for a falling-rate period when the material is no longer completely covered with moisture. High-intensity air-borne ultrasound introduces pressure variations at gas/liquid interfaces, and therefore increases the evaporation rate of moisture. During the negative phase of the pressure cycle the moisture is removed and it does not re-enter during the positive pressure phase. This mechanism is more efficient in the constant-rate period of drying when the surface of the material is completely covered with moisture. In a forced-air drying system, the effect of the air velocity is to influence the heat and mass transfer. The acoustic energy produces an oscillating-velocity effect which can increase the drying rate at stable air velocity. In addition, high-intensity air-borne ultrasound causes microstreaming at the interfaces. This reduces the diffusion boundary layer, increases mass transfer and accelerates diffusion [4]. Therefore, the application of air-borne acoustic energy can positively contribute in the drying process. Nevertheless, the use of this technique has been very limited, probably because of the insufficient improvements obtained in the drying rates and/or the practical difficulties to meet large-scale needs of industry.

When high-intensity ultrasonic energy is directly coupled to the material to be dried, it travels through the solid medium causing a rapid series of alternative contractions and expansions, in a similar way to a sponge when it is squeezed and released repeatedly (sponge effect). The alternating stress makes dewatering easy by creating microscopic channels for water movement. In addition, high-intensity ultrasound produces cavitation (cavity formation, expansion and/or implosion) which may be beneficial for the removal of moisture strongly attached. [5]

During many years we have been involved in the study and development of a new technology for high-intensity sonic and ultrasonic applications in fluids and in multiphase media. This technology is based on a new type of ultrasonic power generator which implements high power capacity, efficiency and directivity. 
This paper deals with the application of the new power ultrasound technology to vegetable dehydration. Two experimental studies are presented: forced-air drying assisted by air-borne ultrasound and a new drying procedure in which the ultrasonic vibration is directly applied to the material together with a static pressure.

\section{THE NEW POWER ULTRASOUND TECHNOLOGY}

For the generation and application of high-intensity ultrasound to different media special high power transducers are needed covering adequate requirements. Particularly, the fluid media (specially the gases) present a low specific acoustic impedance and a high acoustic absorption. Therefore, in order to obtain an efficient transmission of energy, it is necessary to achieve the following requirements: a good impedance matching between the transducer and the medium, large amplitude of vibration and high-directional or focused beams for energy concentration. In addition, for large-scale industrial applications, high power capacity and extensive radiating area would be required in the transducers. At present, most high-power commercial transducers are based on the classical sandwich transducer. They generally consist of a piezoelectric element of transduction in a sandwich arrangement and one or several transmission lines, called mechanical amplifiers or horns, formed by half wavelength resonant metallic elements vibrating extensionally. This kind of transducers have many limitations to cover the above-mentioned requirements. In particular, they are very limited for industrial uses because of their small radiating area.

Therefore new transducer technology is needed where the main points to be considered for successful applications are the increase in power capacity and efficiency, the enlargement of the working area and the improvement of the radiator design to reach, as much as possible, a uniform distribution of vibration amplitude.

During many years we have been involved in the development of a new type of ultrasonic power generator for use in fluids and in multiphase media which implements high power capacity, efficiency, directivity and extensive radiating area. 
The new sonic/ultrasonic power generator is constituted by a transducer with a flexural-vibrating plate radiator and an electronic unit for driving the transducer. The basic structure of the transducer is shown in Fig. 1.

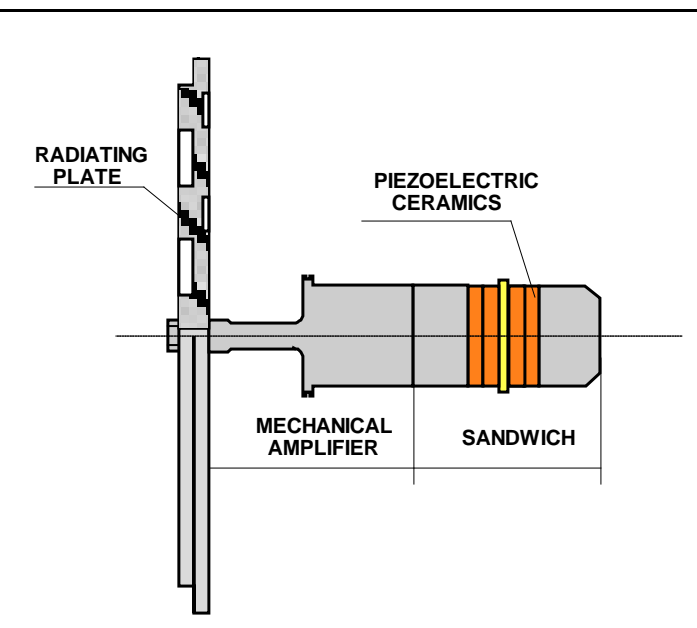

FIGURE 1: Structure of the stepped-plate transducer

It consists essentially of an extensive vibrating plate of stepped profile driven by a piezoelectrically activated vibrator. The vibrator itself consists of a piezoelectric element of transduction in a sandwich configuration and a solid horn which acts as a vibration amplifier. The extensional vibration generated by the transducer element and amplified by the mechanical amplifier, drives the radiating plate which vibrates flexurally in one of its modes. The extensive surface of the plate increases the radiation resistance and offers the vibrating system good impedance matching with the medium. In addition, the special profile of the plate permits the control of the vibration distribution and of the radiation pattern in such a way that a rather uniform vibration amplitude and a high directional or focused radiation can be obtained in order to produce high-intensity acoustic levels. The idea behind the design of the stepped profile is as follows. A flat plate radiator presents in general a poor directivity pattern due to phase cancellation. Nevertheless, if the surface elements vibrating in counterphase on the two side of the nodal lines are alternatively shifted along the acoustic axis to half a wavelength of the sound in the propagation medium, the radiation produced will be in phase across the whole beam and a directivity pattern equivalent to that of the theoretical piston will be obtained.

Following a similar procedure it is possible, with adequate displacements of the different plate zones, to achieve any acoustic field configuration. Focused radiators were also designed and constructed.

The stepped structure of the radiators permits the control of mass distribution along the plate in order to obtain a rather uniform vibration pattern. 
Different prototypes of stepped-plate directional and focused transducers were developed for the frequency range 10-40 kHz and power capacities between $100 \mathrm{~W}$ (20 cm plate diameter) and $1 \mathrm{~kW}$ (70 cm plate diameter) [6, 7, 8 ,9] In the last version of the directional type transducer, beamwidths (at $3 \mathrm{~dB}$ ) of $1.5^{\circ}$ and efficiencies of about $80 \%$ were attained. Presently, we are developing a new model with one meter plate and an estimated power capacity of about $3 \mathrm{~kW}$.

The electronic system to drive the transducer was designed with the aim of producing a signal lying during all the working time within the very narrow band corresponding to the resonance frequency of the transducer. Because this frequency can slide during operation in association with changes in the mechanical load, the electronic circuit has to be able to automatically correct the frequency of the generated signal. The procedure applied was to keep in phase the voltage and current signals in the transducer, following the principle that piezoelectric transducers provide a purely resistive electric impedance at resonance (after compensation of the interelectrode capacitance) [10].

\section{FORCED-AIR DEHYDRATION ASSISTED BY AIR-BORNE ULTRASOUND}

The application of air-borne ultrasonic energy for drying materials has been explored for several decades. Nevertheless, a few air-coupled ultrasonic dryer were reported in the technical literature and apparently none of them was used in large-scale industrial plants The main difficulty, apart from the generation problems, is the transfer of acoustic energy from air into the product due to the acoustic impedance mismatch. Then the major effect is produced at the liquid/gas interface.

We have applied the new stepped-plate ultrasonic generators, as air-borne radiators, in combination with forced air at different temperatures, in order to quantify the effect of acoustic drying, coupling the energy through the atmosphere. 


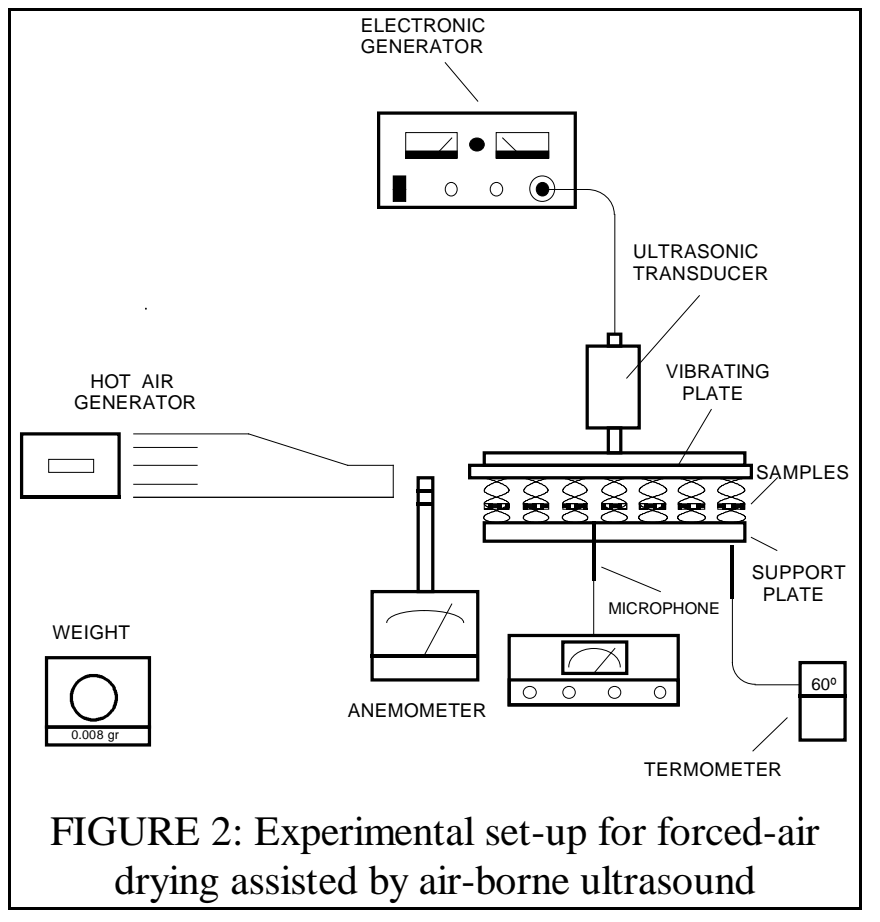

The experimental set-up designed and constructed for airborne ultrasonic dehydration is shown in Fig. 2. It mainly consists of a hot- air generator, a stepped-plate power ultrasonic transducer with the corresponding electronic generator and a flat plate parallel to the ultrasonic radiator, acting as a reflector for the formation of a standing wave and also as sample holder. In addition, complementary sets of equipment for measuring temperature, air flow velocity and weight were used. The acoustic field was previously measured and then controlled through the values of the applied current and voltage.
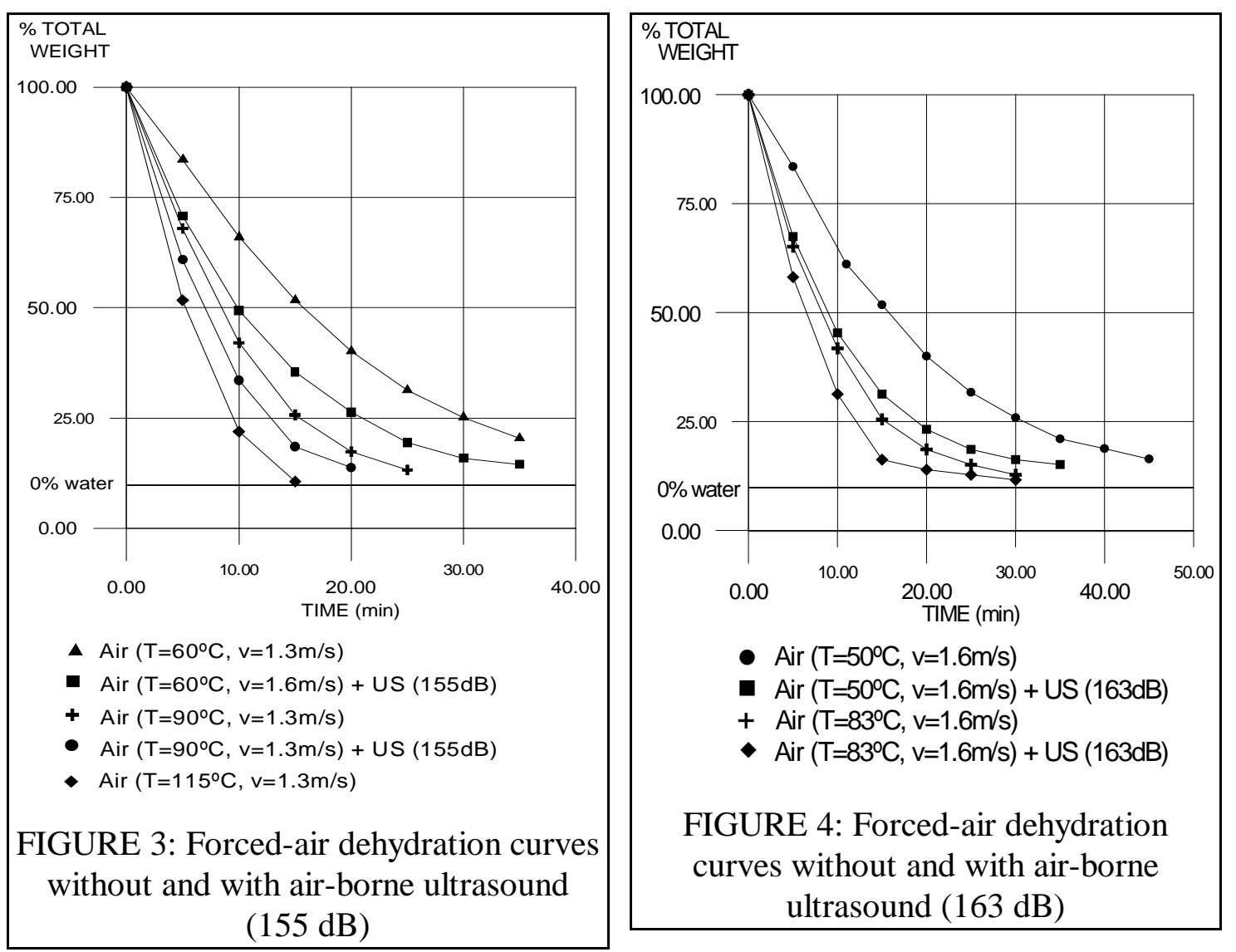
The experimental tests mainly consisted in measuring the water content of food samples after different times of application of high-intensity ultrasonic fields in combination with forced-air at various temperatures and flow velocities. In all experiments, the frequency was kept constant at about $20 \mathrm{kHz}$, while different sound pressure levels were applied. The water content of the samples was measured by weighting them. The food samples to be dried were carrot slices. The slices were either of square section (12x12 mm) or of circular section (14 mm in diameter) with 2, 4 and 8 $\mathrm{mm}$ in thickness.

A summary of the results is presented in Figs. 3 and 4. Fig. 3 shows the results obtained with forced-air at $60^{\circ} \mathrm{C}, 90^{\circ} \mathrm{C}$ and $115{ }^{\circ} \mathrm{C}$ without and with ultrasound (155 $\mathrm{dB})$. In all cases the size of the samples was $12 \times 12 \mathrm{~mm}$ square section with $2 \mathrm{~mm}$ in thickness. As it can be seen, the effect of the ultrasonic radiation is notable at low air temperature and is diminishing when temperature increases. At the highest temperature $\left(115^{\circ} \mathrm{C}\right)$ the ultrasonic effect was negligible and there were no appreciable differences in the curves.

In order to see the influence of the acoustic pressure level some tests were done at higher intensity $(163 \mathrm{~dB})$. Fig. 4 summarises the results of these tests. Comparison between Figs. 3 and 4 shows that the effect of increasing the acoustic pressure is scarcely appreciable and occurs mostly at the falling rate where moisture loses rapidly (i.e., the first 10 minutes), and diminishes as the drying curves start to level off.

Other tests were done by increasing the air flow velocity up to 3 $\mathrm{m} / \mathrm{s}$. The results obtained showed that 
at this forced-air speed the differences with and without ultrasound are less significant (Fig. 5).

In conclusion, it can be stated from the results that the application of air-borne ultrasound can be useful in increasing the efficiency of forced-air drying processes. Nevertheless, the improvement seems to be relatively limited and the use of air-borne ultrasound could be restricted to specific products and/or operations such as heatsensitive materials and/or applications where rapid drying is required.

\section{DEHYDRATION BY DIRECTLY COUPLED ULTRASONIC VIBRATION}

The main difficulty in dehydration by air-borne ultrasonic radiation is the low penetration of the acoustic energy in the food material due to the mismatch between acoustic impedances. In fact, the effectiveness of energy transfer between two media very much depends on their acoustic impedances, defined as the product of density and sound speed in the material.

Therefore, in order to increase the ultrasonic effect on food dehydration a new procedure was developed and tested in which the ultrasonic vibration was applied in direct contact with the food samples and together with a static pressure. To that purpose, the experimental set-up presented in Fig. 2 was modified. The samples were placed on the surface of the transducer radiating plate and they were kept there during treatment by applying a static pressure on them (Fig. 6). An air flow at $1 \mathrm{~m} / \mathrm{s}$ and $22^{\circ} \mathrm{C}$ was also applied to facilitate the removal of moisture. The drying effect was measured following the previous procedure, i.e., by weighting the samples at different times during treatment. Fig. 7 shows the results obtained with carrot slices of 2, 4 and $8 \mathrm{~mm}$ in thickness and 14 $\mathrm{mm}$ in diameter. It can be appreciated that the drying effect was remarkably improved. In fact, the drying process is not only much quicker and less energy consuming than the forced-air drying (with and without air-borne ultrasound) but it is more powerful: the 
final moisture content could be less than $1 \%$. In addition, due to the processing time and low temperature of the air flow, the product qualities are well preserved.

In conclusion, the application of ultrasonic vibration in direct contact with food samples has demonstrated to be very effective method for food

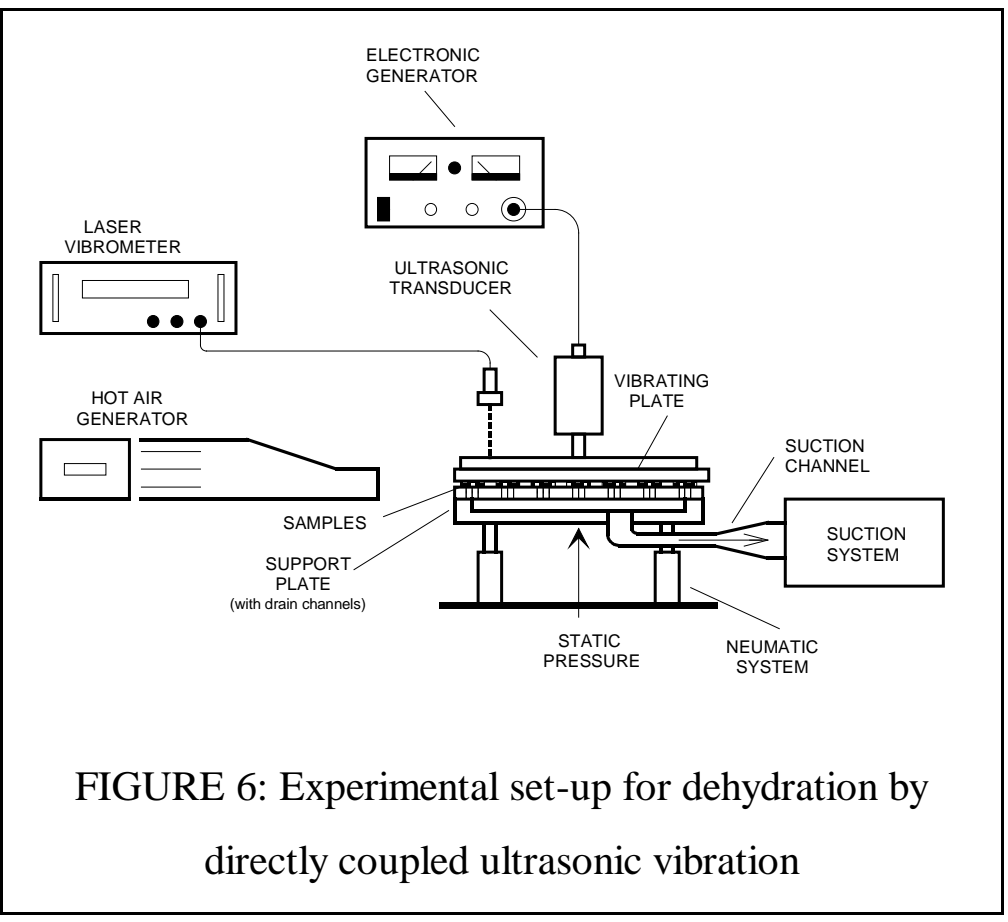
dehydration. The good acoustic impedance matching between the transducer vibrating

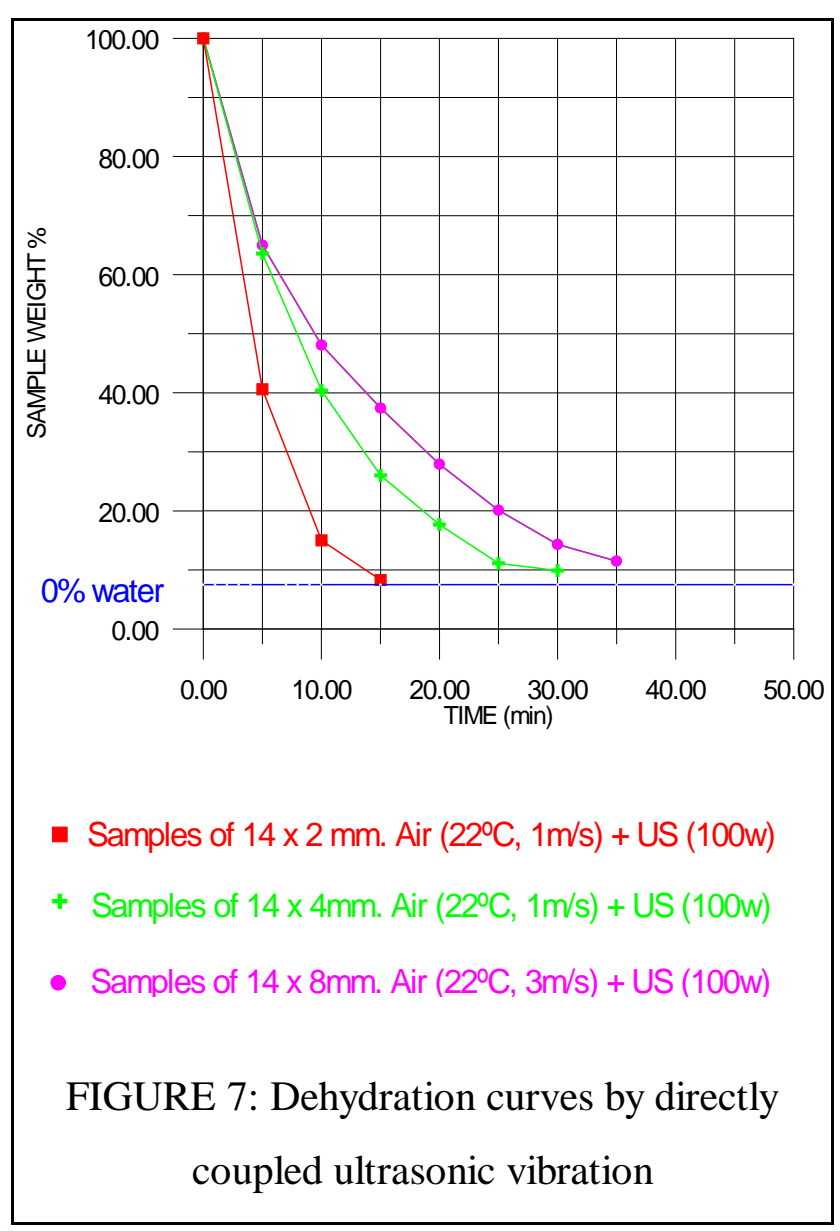
plate and the food material favours the deep penetration of acoustic energy and increased the effectiveness of the process. In fact, the material is subjected to high ultrasonic stresses which, due to the rapid series of contractions and expansions, produce a kind of "sponge effect" and the quick migration of moisture through natural channels or other channels created by the wave propagation. In addition, the production of cavitation may explain the removal of the final percentage of moisture (under 3\%) which generally is strongly attached. 


\section{CONCLUSION}

From the investigation presented in this paper the following conclusions can be stated:

- High-intensity air-borne ultrasound accelerates forced-air drying processes, but the effect is limited. Therefore, this process may be considered useful only to very specific applications such as to dry materials well matched acoustically to air or to assist in operations where hot-air at moderate temperature is needed to preserve the product.

- The application of ultrasonic energy directly coupled to the food samples by using the stepped-plate transducers has demonstrated to be very effective method for food dehydration. The main advantages of this process are the optimum energy transfer and the capability for industrial large-scale applications because of the extensive surface of the plate radiators.

\section{REFERENCES}

1. Fairbank, H.V, 1975. Applying ultrasound to continuous drying process. Ultrasonic International 1975 Conference Proceedings, IPC Science and Technology Press Ltd, Guildford, UK, pp. 43-45.

2. Seya, K, 1970. Macrosonic drying. Proceedings of the First International Symposium on High-power Ultrasonics, IPC Science and Technology Press Ltd, Guildford, UK, pp. 136-140.

3. Soloff, R.S., 1994. Sonic Drying, J. Acoust. Soc. Am. 36: 961-965.

4. Arkhangel'skii, M. F. And Statnikov, Y.G., 1973. Diffusion in heterogeneous systems in: Physical Principles of Ultrasonic Technology, Vo. 2 , L.D. Rosemberg, Plenum Press, New York. 
5. Muralidhara, H. S., Ensminger, D. and Putman, A., 1985. Acoustic dewatering and drying (low and high frequency): State of the Art review. Drying Technology 3: 529566.

6. Gallego-Juárez, J. A., Rodriguez-Corral, G., Gaete-Garretón, L. ,1978. Ultrasonics, (16) 6: 267-271.

7. Gallego-Juárez, J. A., Rodríguez-Corral, G. Montero de Espinosa,, F., Andrés Gallegos, E., San Emeterio-Prieto, J.L, 1983. Proceedings Ultrasonic International, Butterworth Co. Ltd., UK, 295-300

8. San Emeterio-Prieto, J. L., Gallego-Juárez, J. A., Rodríguez Corral, G., 1987. Journal of Sound and Vibration, (14) 3: 495-505.

9. Rodríguez-Corral, G., San Emeterio-Prieto, J.L., Gallego-Juárez, J. A., 1987. Proceedings Ultrasonic International, Butterworth Co. Ltd., UK, 794-799.

10.Gallego-Juárez, J. A., Rodriguez-Corral, G., San Emeterio-Prieto, J. L., MontoyaVitini, F., 1994. Electroacoustic unit for generating high sonic and ultrasonic intensities in gases and interphases, US Patent $N^{\circ}$ 5,299,175. 